\title{
De la formation à la pratique professionnelle en psycho-oncologie
}
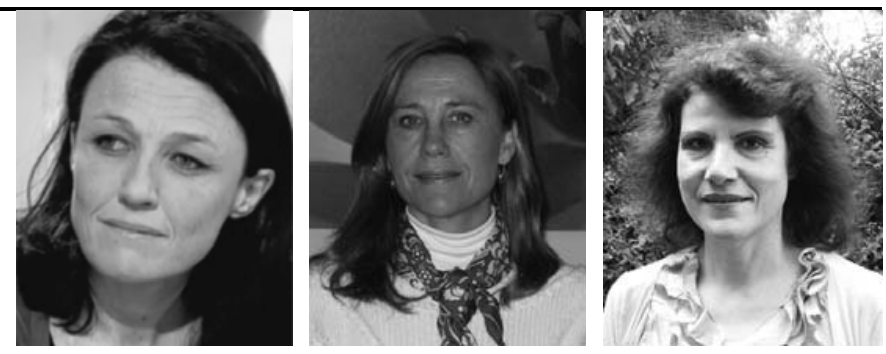

\section{From Training to Professional Practice in Psycho-Oncology}

\author{
S. Dauchy - F. Ellien - M.-F. Bacqué \\ (C) Springer-Verlag France 2014
}

Le XXX ${ }^{\mathrm{e}}$ Congrès de la SFPO (Société Française de PsychoOncologie) en décembre 2013, s'est donné pour thème la formation, celle des psychologues et des psychiatres comme celle de l'ensemble des acteurs de soins, pour que soient mieux pris en compte les enjeux psychiques dans la prise en charge globale des malades. Le Plan cancer 2014-2019 est venu confirmer l'importance majeure de cette thématique, en rappelant la nécessité de formations à une meilleure prise en charge, notamment en soins de support (mesure 4.5), mais aussi à la communication (pour les oncologues, mesure 4.4), ou encore au repérage des besoins et de la vulnérabilité (pour tous, mesure 7.3). Comme l'attendait la SFPO, l'offre de soins psychologiques, avec celle des soins de support, devient une exigence qualitative et quantitative de l'agrément des établissements à la pratique de la cancérologie (mesure 7.6), un des objectifs étant de permettre l'intégration effective dans les programmes personnalisés de soins comme de l'après-cancer de la dimension psychologique et sociale.

\footnotetext{
S. Dauchy $(\bowtie)$

Gustave-Roussy

114, rue Édouard-Vaillant,

F-94805 Villejuif, France

e-mail : Sarah.DAUCHY@gustaveroussy.fr

F. Ellien $(\bowtie)$

Directrice du Réseau de santé SPES,

F-91750 Champcueil, France

e-mail : fellien.spes@wanadoo.fr

M.-F. Bacqué $(\bowtie)$

Rédactrice en chef de Psycho-Oncologie,

EA 3071, université de Strasbourg,

France

e-mail : mfbacque@club-internet.fr
}

\section{Un congrès anniversaire ouvrant sur de nouvelles recommandations, sur la formation des professionnels de la cancérologie, acteurs de soins psychiques mais aussi médecins, soignants et même non-soignants}

Ce congrès anniversaire a ainsi permis de faire un bilan des principales avancées dans le domaine des soins psychiques en cancérologie, dans leur dynamique positive comme dans leurs limites - à l'instar des développements récents de la cancérologie, où l'amélioration des perspectives thérapeutiques et de l'espérance de vie, le développement des soins extrahospitaliers et des traitements oraux ne sont que la face positive d'une discipline marquée à l'inverse par la raréfaction des soins de proximité, la complexité des échanges multidisciplinaires, les temps d'échange et de relation toujours plus courts et contraints... Trente ans après le premier congrès de la SFPO, il nous a permis d'entendre avec bonheur représentants des tutelles, cancérologues et patients témoigner de l'importance de la place aujourd'hui en cancérologie des professionnels de soins psychiques, et de la nécessité de maintenir notre engagement pour le développement d'une prise en charge réellement personnalisée de l'individu, où la personnalisation subjective rejoigne la personnalisation biologique. Il a permis de rappeler certains des grands enjeux de la cancérologie de demain, autour notamment de l'observance, du développement de la médecine prédictive, mais aussi des chimiothérapies ciblées et des traitements oraux. Avec plus de dix communications consacrées à la formation des professionnels, notamment à la communication ou à la prévention des risques psychosociaux, avec la présence de personnalités de la psycho-oncologie francophone très impliquées dans ces enjeux de formation (en particulier les Prs Lise Fillion [Québec], Patrice Guex et Frédéric Stiefel [Lausanne], Darius Razavi [Bruxelles]), ce 
$\mathrm{XXX}^{\mathrm{e}}$ Congrès a permis comme traditionnellement maintenant la rédaction des Recommandations de la Société Française de Psycho-Oncologie pour l'intégration de la psychooncologie dans la formation des acteurs de soins en cancérologie. Elles ont pour objectif notamment de clarifier les critères de qualité d'une formation initiale et continue adaptée pour les psychologues et les psychiatres dont nous attendons la participation toujours plus importante aux prises en charge globales en cancérologie. À côté de celles-ci, les articles présentés ci-après permettront au lecteur d'avoir un accès approfondi aux travaux de certains des orateurs présents. Rappelons sur ce point que l'ensemble des communications et abstracts est disponible sur le site de la SFPO (www.sfpo.fr).

\section{Des patients qui stimulent la formation des professionnels de l'oncologie}

Signalons la présence croissante, dans les congrès de la SFPO, de patients, de proches, de membres d'associations de patients. Leurs interventions sont riches d'initiatives et surtout de témoignages qui font avancer la pratique. Ainsi, Catherine Chayenko Cerisey a présenté, en tant que patiente et bloggeuse, sa vision du patient (qui bien souvent est une patiente) « hors les murs » de l'hôpital, rivé coûte que coûte à son ordinateur. Le « Web social» est pour elle une nouvelle donnée depuis dix ans. Sa permanence, son réseau de personnes disponibles pour soutenir, ses échanges d'informations et de « trucs » pour survivre permettent de supporter l'isolement quand les services répondent aux abonnés absents. Cependant, même si l'anonymat est rompu grâce aux rencontres IRL (in real life), les consultations psychologiques sont recherchées pour tracer cette continuité qui manque sévèrement dans l'hospitalisation et ses suites. Outre l'annonce, la rémission, voire la guérison doivent être accompagnées sur le plan psychologique. Comme pour la rechute ou la fin de vie, le soutien psychologique semble une requête justifiée car le Net ne peut pas tout, surtout chez des femmes qui se sont fixées pour défi de tout réussir, l'annonce du cancer, la charge de la maladie, le retour au travail, la réhabilitation ; en maintenant de surcroît une sexualité épanouie et des enfants à l'aise avec la maladie de leur parent.

\section{Psychologues et psychiatres entre demande et désir ?}

Cet idéal de la prise en charge, les psychologues et les psychiatres le connaissent bien, eux qui sont partagés entre puissance et démunition face au cancer. Dans une optique résolument psychanalytique, Caroline Doucet présente les principes permettant de ne pas céder devant ces deux écueils. La question du sens du symptôme et du sens de la maladie ne peut qu'être déduite de ce qu'en dit le malade et non observé de l'extérieur par le clinicien. Comme Caroline Doucet, Sophie Claris reprend la question du transfert sur le clinicien qui transite par celui de l'institution et du médecin et questionne ce que reclament les services lorsqu'ils demandent l'intervention de l'« oncopsy ». Le travail du psychologue redonne place au singulier dans une expérience presque banale aujourd'hui. Mais malgré l'augmentation des cancers, chaque personne élabore sa propre intégration de la maladie dans son parcours de vie et y gagne ainsi une forme d'autonomie. Psychologues et psychiatres facilitent ce temps gagné pour l'existence quelle que soit l'issue de la maladie. Insistons également sur l'effet du soutien ou de la psychothérapie sur l'ensemble des proches. Le mieux-être d'un patient fait en effet gagner à toute sa famille et ses amis une amélioration considérable, on n'insistera jamais assez sur les conséquences d'un deuil non préparé ou traumatique sur la filiation.

\section{Psychologues et médecins en oncologie peuvent être aidés par le groupe Balint}

Les groupes Balint, dénommés par Michaël Balint «groupes de formation-recherche ", permettent cette réflexion en parallèle entre un membre du groupe qui expose une situation « en cours » et ses collègues. La « situation » résulte, en réalité, du suivi d'un malade par un soignant ou un médecin ou de la psychothérapie d'un psychologue. Les membres du groupe Balint qui se retrouvent régulièrement vont travailler ce cas, bien qu'ils n'en soient pas responsables. Le dispositif donne donc à tous, selon Alice Polomeni, la possibilité de s'entendre spontanément rapporter son expérience subjective d'une relation avec un patient, et en même temps, d'écouter les autres apporter leur « cas » à leur tour. Le système $\mathrm{a}$ fait ses preuves depuis les années 1960. Il est en place dans de nombreuses facultés de médecine et de psychologie, hélas encore trop peu en France.

\section{Une formation à la psychologie hétérogène chez les médecins}

Marjorie Roques et Nadine Proia-Lelouey déplorent une situation hétérogène dans la formation en psychologie des médecins français. Elles plaident pour une formation générale descriptive des mécanismes psychiques, mais surtout pour une connaissance approfondie des effets inconscients de la maladie sur les patients et sur les soignants. L'approche double mêlant enseignement et mise à contribution dans les jeux de rôles, les supervisions de groupe ou individuelles permettraient une plus grande satisfaction des malades, mais aussi une approche moins centrée sur la performance et plus tournée vers l'humain. 


\section{Empathie et théorie de l'esprit}

Ralph Balez et al. questionnent à ce sujet le concept d'empathie pour les étudiants en médecine. Dans le cursus du médecin d'aujourd'hui, la « compétence en communication » est recommandée comme une véritable panacée : elle améliorerait les résultats des traitements au travers de l'adhésion aux soins, de la satisfaction des traitements. Or, l'empathie est bien plus qu'une compétence en communication. Elle renvoie aujourd'hui à la théorie de l'esprit. Il s'agit de comprendre les émotions et les processus mentaux de soi-même et d'autrui, de façon à donner du sens à ses réactions. L'empathie engendrerait l'eumétrie, cette capacité à trouver la bonne distance entre individus, pour une relation satisfaisante entre eux. L'empathie du médecin à l'égard de ses patients améliorerait aussi le bien-être du praticien, une meilleure orientation parmi les spécialités médicales et limiterait l'épuisement professionnel... Malheureusement, de nombreuses recherches montraient que la capacité du médecin à être empathique avec ses patients diminuait au cours des études. Or, avec une démarche pédagogique propre à la faculté de médecine de Brest, il a été possible (sur 63 étudiants) d'augmenter au contraire cette aptitude. La formation emploie des films d'une consultation avec acteurs, des jeux de rôles des médecins, un travail de groupe, puis une simulation d'annonce d'un lymphome entre médecins (jouant patient et médecin). Si l'empathie augmente immédiatement après la formation, six mois plus tard, les médecins observent qualitativement qu'ils ont bénéficié de la formation. Il est nécessaire de poursuivre cette recherche pour en consolider les résultats, mais l'optimisme peut être de mise pour l'amélioration de l'approche psychologique des patients par les médecins.

\section{Dépister la détresse des patients favorise la communication entre soignants}

Au Québec, Lise Fillion et ses collaboratrices développent des outils pour dépister la détresse chez les patients, et en profitent aussi pour augmenter la collaboration interprofessionnelle, dans un pays où le rôle des infirmières spécialisées dans sa prise en charge psychologique est beaucoup plus développé qu'en France. Rien de tel en effet pour remotiver les équipes et leur donner l'occasion d'être actives pour changer. C'est exactement ce que constatent Nena Stadelmaier et ses collègues : leur guide d'entretien (PO-Bado) permet de repérer les difficultés psychosociales liées à l'annonce, mais dépasse le simple dépistage par la mise en place d'une relation d'aide. Il modifie les pratiques des soignants vis-à-vis des patients bien sûr (en limitant les questions qui ferment la communication ou en proposant une orientation vers les psychistes), mais aussi entre eux. La posture relationnelle des soignants ainsi adoptée se fixe sur le vécu subjectif du patient en " osant » prendre le " risque » de questions plus globales, décentrées des symptômes.

Les questions de l'observance, de l'alliance thérapeutique, de la communication patient-soignant, de la prise en charge pluridisciplinaire, de l'impact psychique des nouvelles organisations font donc partie des nouveaux défis de la formation... Ce numéro montre l'extraordinaire créativité des différents acteurs du soin du cancer comme du soin psychique. La formation des professionnels du soin doit mettre à contribution tous les partenaires de l'oncologie mais aussi les spécialistes de la pédagogie, des sciences de l'éducation et de la psychologie des organisations pour élaborer des programmes dynamiques, employant les outils les plus interactifs (mise en situation, arts visuels, théâtre) et rendant vivantes les remises en cause salutaires des savoirs et des pratiques. 